\title{
Universal finite-size scaling amplitudes in anisotropic scaling
}

\author{
Malte Henkel $^{a}$ and Ulrich Schollwöck ${ }^{b}$ \\ ${ }^{a}$ Laboratoire de Physique des Matériaux, ${ }^{*}$ Université Henri Poincaré Nancy I, \\ B. P. 239, F-54506 Vandouvre-les-Nancy Cedex, France \\ ${ }^{b}$ Sektion Physik, Ludwig - Maximilians -Universität München, Theresienstr. 37/III, D-80333 München, Germany
}

\begin{abstract}
Phenomenological scaling arguments suggest the existence of universal amplitudes in the finitesize scaling of certain correlation lengths in strongly anisotropic or dynamical phase transitions. For equilibrium systems, provided that translation invariance and hyperscaling are valid, the PrivmanFisher scaling form of isotropic equilibrium phase transitions is readily generalized. For nonequilibrium systems, universality is shown analytically for directed percolation and is tested numerically in the annihilation-coagulation model and in the pair contact process with diffusion. In these models, for both periodic and free boundary conditions, the universality of the finite-size scaling amplitude of the leading relaxation time is checked. Amplitude universality reveals strong transient effects along the active-inactive transition line in the pair contact process.
\end{abstract}

64.60.Ht, 05.70Fh, 82.20Db

\section{INTRODUCTION}

The notions of scaling and universality are central to the modern understanding of critical phenomena, see e.g. [1] 3. Besides the well-known universality of the critical exponents, universality is also manifest for many critical amplitudes, as reviewed in . 1 . Here we are concerned with universal amplitudes which arise in finite-size scaling. For example, consider a statistical system at equilibrium, e.g. a simple ferromagnet, which is described by an isotropic and translation-invariant field theory in its continuum limit. Close to its critical point, and on a lattice with finite extent $L$, Privman and Fisher [5] showed that in any dimension $d$ below the upper critical dimension $d^{*}$ the singular part of the free energy density $f$ and the inverse correlation lengths $\xi_{i}$ satisfy the scaling form

$$
f(t, h)=L^{-d} Y\left(z_{1}, z_{2}\right), \xi_{i}^{-1}=L^{-1} S_{i}\left(z_{1}, z_{2}\right),
$$

where $z_{1}=C_{1} t L^{1 / \nu}, z_{2}=C_{2} h L^{(\beta+\gamma) / \nu}$ are the scaling variables, the reduced temperature $t=\left(T-T_{c}\right) / T_{c}$ and the reduced magnetic field $h=H / T_{c}$ describe the distance from the critical point and $\beta, \gamma, \nu$ are the standard equilibrium critical exponents. The index $i$ distinguishes different correlation lengths, e.g. $i=\sigma$ for the spin-spin correlation length or $i=\varepsilon$ for the energy-energy correlation length in a simple ferromagnet. Furthermore, the scaling functions $Y$ and $S_{i}$ are universal functions and all non-universal properties of a given model are condensed in the values of the non-universal metric factors $C_{1}$ and $C_{2}$. Although the functions $Y, S_{i}$ are universal, they do depend on the boundary conditions and of the geometric shape of the lattices under study.

It follows from (11) that if the model parameters are tuned to $t=h=0$ so that the model is at its bulk critical point, the finite-size scaling of free energy density $f=L^{-d} Y(0,0)$ and of the inverse correlation lengths $\xi_{i}^{-1}=L^{-1} S_{i}(0,0)$ is described in terms of universal finite-size scaling amplitudes. This qualitative statement can be made quantitative in $2 D$ for systems defined on an infinitely long strip of finite width $L$ through a by now classic conformal invariance argument which for example explicitly relates the $S_{i}(0,0)$ to known critical exponents [6]. Concrete model studies have confirmed this many times, as reviewed in 3, A. More recently, similar relations have been conjectured also in $3 D$ from the results of numerical studies in toruslike [7,8] and spherical geometries [9, 10].

When considering the situation of dynamical scaling, where time and space scale differently, the extra degree of freedom might appear to exclude the presence of universal finite-size scaling ampltitudes in the sense of eq. (11). Here, we shall ask under what conditions the arguments of Privman and Fisher [5] can be generalised also to anisotropic scaling. Specifically, for a system undergoing a phase transition with anisotropic scaling which is defined on a lattice with finite extent $L$ in the spatial direction but is infinite in the temporal direction. Then (under conditions to be detailed in sections 2 and 3 ) the spatial correlation lengths should satisfy the scaling form

$$
\xi_{i, \perp}^{-1}=L^{-1} S_{i}\left(C_{1} t L^{1 / \nu_{\perp}}, C_{2} h L^{(\beta+\gamma) / \nu_{\perp}}\right)
$$

in a notation analogous to (11) and where again the $S_{i}$ are universal functions. 
While the generalization to equilibrium anisotropic scaling is a rather straightforward extension of the methods valid for the isotropic case [5], the non-equilibrium situation is more difficult. We shall show how correlation length amplitude universality can be established for systems in the directed percolation universality class. Furthermore, numerical data from some reaction-diffusion models are also in agreement with this and suggest that the scaling form (2) might be generally valid.

This paper is organised as follows: in section 2, we present the scaling arguments leading to the recognition of universal amplitudes for anisotropic scaling in equilibrium. In section 3 non-equilibrium dynamical scaling is discussed, with emphasis on the directed percolation universality class. In section 4 , we test the amplitude universality by analysing the finite-size scaling of the leading inverse relaxation time in the annihilation-coagulation model and the pair contact process with single particle diffusion. In section 5 we give our conclusions. An appendix contains the Bethe ansatz calculation of the relaxation time in an analytically solvable special case.

\section{UNIVERSAL FINITE-SIZE AMPLITUDES IN EQUILIBRIUM}

Consider an equilibrium system with anisotropic scaling in two distinct directions. Systems of this kind are known since a long time, e.g. in Lifshitz points [11] or anisotropic uniaxial magnets [12], see [13 16] for reviews or else in quantum phase transitions [17, see [18] for a recent book. One can derive the phenomenological scaling of the physical observables from the covariance of the correlators under scale transformations and reconstruct the thermodynamics this way, see [2, 3]. In line with conventional phenomenological treatments of strongly anisotropic scaling [19,20], we assume for the two-point functions the scaling form

$$
G_{i}\left(r_{\perp}, r_{\|} ; t, h\right)=b^{-2 x_{i}} G_{i}\left(\frac{r_{\perp}}{b}, \frac{r_{\|}}{b^{\theta}} ; t b^{y_{t}}, h b^{y_{h}}\right)
$$

where $b$ is the rescaling factor, $t, h$ refer to physical quantities like the reduced temperature and the reduced magnetic field, $\theta$ is the anisotropy exponent and $y_{t}, y_{h}, x_{i}$ are scaling exponents. The index $i$ refers to different physical quantities of which the two-point function is formed, e.g. $i=\sigma$ for the spin operator or $i=\varepsilon$ for the energy density (for simplicity, we use throughout a notation analogous to simple ferromagnets). At criticality, $t=h=0$, and one has

$$
\begin{aligned}
G_{\sigma}\left(r_{\perp}, 0\right) & \sim r_{\perp}^{-2 x_{\sigma}}, G_{\sigma}\left(0, r_{\|}\right) \sim r_{\|}^{-2 x_{\sigma} / \theta}, \\
G_{\varepsilon}\left(r_{\perp}, 0\right) & \sim r_{\perp}^{-2 x_{\varepsilon}}, G_{\varepsilon}\left(0, r_{\|}\right) \sim r_{\|}^{-2 x_{\varepsilon} / \theta} .
\end{aligned}
$$

For a strongly anisotropic equilibrium system, $r_{\perp}$ and $r_{\|}$correspond to different directions in space. This case is for example realised at the Lifshitz point in spin systems with competing interactions like the ANNNI model [15,21]. For brevity, we shall refer to the directions $r_{\perp}$ as 'spatial' and to the directions $r_{\|}$as 'temporal'.

We use the equilibrium fluctuation-dissipation theorem

$$
\chi=\int \mathrm{d} r_{\|} \mathrm{d}^{d} r_{\perp} G_{\sigma}\left(r_{\perp}, r_{\|}\right), C=\int \mathrm{d} r_{\|} \mathrm{d}^{d} r_{\perp} G_{\varepsilon}\left(r_{\perp}, r_{\|}\right),
$$

where $d$ is the number of 'spatial' dimensions and $\chi, C$ are the susceptibility and specific heat. We shall work with a single 'temporal' direction throughout, but generalisations are obvious. Units are such that the critical temperature $T_{\mathrm{c}}=1$. From (3) and integrating, one gets immediately the scaling forms for $\chi=\chi(t, h)=-\partial^{2} f / \partial h^{2}$ and for $C=C(t, h)=-\partial^{2} f / \partial t^{2}$. Here $f$ is the (singular part) free energy density. From the scaling of $\chi$ and $C$, it should satisfy the scaling

$$
f(t, h)=b^{\theta+d-2 x_{\sigma}-2 y_{h}} f\left(t b^{y_{t}}, h b^{y_{h}}\right)=b^{\theta+d-2 x_{\varepsilon}-2 y_{t}} f\left(t b^{y_{t}}, h b^{y_{h}}\right) .
$$

These two forms are consistent, if $x_{\sigma}+y_{h}=x_{\varepsilon}+y_{t}=w$. In fact, the above argument works for any pair of scaling operators like $\sigma, \varepsilon$ and their conjugated scaling fields $h, t$. Therefore, the value $w$ must be independent of all physical scaling operators which might be present in a given model. Next, we define the standard static critical exponents $\alpha, \beta, \gamma$ as usual, e.g. [2]. Also, out of criticality, we expect an exponential decrease of the two-point function,

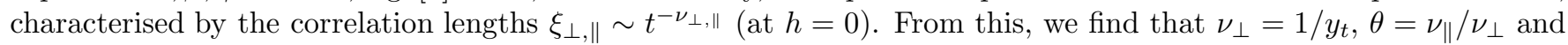
$\beta+\gamma=y_{h} / y_{t}$. From dimensional counting, we expect $w=d+\theta$ and the free energy density then scales as

$$
f(t, h)=b^{-d-\theta} f\left(t b^{y_{t}}, h b^{y_{h}}\right) .
$$


As usual, we explicitly assume the absence of dangerously irrelevant scaling fields [1,5, 19]. We then recover the hyperscaling relation $2-\alpha=\nu_{\|}+d \nu_{\perp}$. The common scaling form for the thermodynamics is found by scaling out $b$ and introducing the conventional critical exponents:

$$
f(t, h)=A_{1}|t|^{2-\alpha} W^{ \pm}\left(A_{2} h|t|^{-\beta-\gamma}\right),
$$

where $W^{ \pm}$are the universal scaling functions which are obtained for $t>0$ and $t<0$, respectively, and $A_{1,2}$ are non-universal metric constants. At this level, the anisotropy of the scaling of the two-point function only appears in the generalized form of the hyperscaling relation.

Equation (8) will be the starting point for our discussion of finite-size scaling. In what follows, we consider a situation where the 'spatial' directions are of finite extent $L$ whereas the 'temporal' direction remains infinite. In the same spirit as Privman and Fisher [5], we assume that the finite-size scaling behaviour is governed by the 'spatial' correlation length $\xi_{\perp}$ only and we write

$$
f(t, h ; L)=A_{1}|t|^{2-\alpha} W^{ \pm}\left(A_{2} h|t|^{-\beta-\gamma} ; L \xi_{\perp}^{-1}\right),
$$

where the bulk 'spatial' correlation length $\xi_{\perp}=\xi_{0} t^{-\nu_{\perp}}$. Note that there is no extra metric factor in the second argument of $W^{ \pm}$, whereas $\xi_{0}$ is non-universal. To simplify the notation, we assume that there is no phase transition for $L$ finite, but this restriction could be removed analogously to the equilibrium case [22]. We emphasize that the 'temporal' direction remains infinite, otherwise we would have to deal with two distinct finite length scales.

Following the ideas developed by Privman and Fisher [5], we have to trace the non-universal constants, taking into account the anisotropic scaling. For that, it is sufficient to study the 'spatially' infinite system. We expect the scaling form of the connected spin-spin correlator, see also (3),

$$
G_{\sigma}\left(r_{\perp}, r_{\|} ; t, h\right)=D_{0} D_{1} r_{\perp}^{-2 x_{\sigma}} X^{ \pm}\left(r_{\perp} / \xi_{\perp}, D_{0} r_{\|} / \xi_{\perp}^{\theta} ; D_{2} h|t|^{-\beta-\gamma}\right),
$$

where $X^{ \pm}$is a universal scaling function and $D_{0,1,2}$ are non-universal metric factors. From the fluctuation-dissipation theorem (可) one has

$$
\chi(t, h)=D_{1} \xi_{\perp}^{\gamma / \nu_{\perp}} \tilde{X}^{ \pm}\left(D_{2} h|t|^{-\beta-\gamma}\right) .
$$

where $\widetilde{X}^{ \pm}$is a new scaling function obtained from $X^{ \pm}$. Now, for the non-connected spin-spin correlator, one has in the same way, introducing new universal scaling functions $Z^{ \pm}$

$$
\Gamma_{\sigma}\left(r_{\perp}, r_{\|} ; t, h\right)=D_{0} D_{1} r_{\perp}^{-2 x_{\sigma}} Z^{ \pm}\left(r_{\perp} / \xi_{\perp}, D_{0} r_{\|} / \xi_{\perp}^{\theta} ; D_{2} h|t|^{-\beta-\gamma}\right)
$$

At this point, we assume translation invariance with respect to $r_{\perp}$ and $r_{\|}$. Therefore, there should exist a mean magnetization $m$ which is independent of $r_{\perp}$ and $r_{\|}$and which can be found considering $\Gamma_{\sigma}$ at large separations $r_{\perp}, r_{\|}:$

$$
\begin{gathered}
\Gamma_{\sigma}\left(r_{\perp}, r_{\|} ; t, h\right)=D_{0} D_{1} \xi_{\perp}^{-2 x_{\sigma}} Z^{ \pm}\left(1, D_{0} r_{\|} / r_{\perp}^{\theta} ; D_{2} h|t|^{-\beta-\gamma}\right) \\
\downarrow \\
\downarrow \\
m^{2}(t, h)=D_{0} D_{1} \xi_{\perp}^{-2 x_{\sigma}} \widetilde{Z}^{ \pm}\left(D_{2} h|t|^{-\beta-\gamma}\right),
\end{gathered}
$$

where the arrow indicates taking the limit of large 'spatio-temporal' separations. Because of translation invariance, $m^{2}$ should become independent of $D_{0}$. On the other hand, applying standard thermodynamics to the free energy (8) yields

$$
\begin{aligned}
m(t, h) & =A_{1} A_{2}|t|^{\beta} W_{1}^{ \pm}\left(A_{2} h|t|^{-\beta-\gamma}\right), \\
\chi(t, h) & =A_{1} A_{2}^{2}|t|^{-\gamma} W_{2}^{ \pm}\left(A_{2} h|t|^{-\beta-\gamma}\right),
\end{aligned}
$$

where $W_{n}^{ \pm}(x)=\mathrm{d}^{n} W^{ \pm}(x) / \mathrm{d} x^{n}$.

We now compare eqs. (11) and (16). Letting first $h=0$, we find

$$
D_{1} \xi_{0}^{\gamma / \nu_{\perp}}=A_{1} A_{2}^{2} U_{1}
$$

Comparing the arguments of the scaling functions, we have 


$$
D_{2}=A_{2} U_{2} .
$$

Next, we compare eqs. (14) and (15) and find for $h=0$ that

$$
D_{0} D_{1} \xi_{0}^{-2 \beta / \nu_{\perp}}=A_{1}^{2} A_{2}^{2} U_{3}
$$

(since $\left.x_{\sigma}=\beta / \nu_{\perp}\right)$. Here, $U_{1,2,3}$ are universal constants whose universality follows from the universality of the scaling functions considered. Using the hyperscaling relation $\gamma+2 \beta=(d+\theta) \nu_{\perp}$, we find that

$$
\begin{aligned}
A_{1} \xi_{0}^{d+\theta} D_{0}^{-1} & =Q_{1}=U_{1} / U_{3} \\
D_{2} A_{2}^{-1} & =Q_{2}=U_{2} \\
D_{0}^{\gamma /\left(\nu_{\perp}(d+\theta)\right)} D_{1} A_{1}^{-1-\gamma /\left(\nu_{\perp}(d+\theta)\right)} A_{2}^{-2} & =Q_{3}=U_{1}^{1-\gamma /\left(\nu_{\perp}(d+\theta)\right)} U_{3}^{\gamma /\left(\nu_{\perp}(d+\theta)\right)}
\end{aligned}
$$

and the $Q_{1,2,3}$ are universal constants.

Finally, we come back to the finite-size scaling behaviour. In eq. (9), we replace $\xi_{0}$ by $A_{1}$ using (20). Scaling out $L$ and using again hyperscaling, it is easy to arrive at the scaling form

$$
f(t, h ; L)=L^{-d-\theta} D_{0} Y\left(C_{1} t L^{1 / \nu_{\perp}}, C_{2} h L^{(\beta+\gamma) / \nu_{\perp}}\right),
$$

where $Y$ is a universal scaling function and $C_{1,2}$ are non-universal metric factors related to $A_{1,2}$. In contrast with the isotropic situation (1]), we see that the finite-size scaling amplitude of the free energy is no longer universal. Furthermore, since $\xi_{\|}=\xi_{\perp}^{\theta} / D_{0}$, we have

$$
f(t, 0 ; L) \xi_{\perp}^{d}(t, 0 ; L) \xi_{\|}(t, 0 ; L)=D_{0}^{-1} f(t, 0 ; L) \xi_{\perp}^{d+\theta}(t, 0 ; L) \underset{t \rightarrow 0}{\rightarrow} \text { univ. constant, }
$$

which holds because of (20). Therefore, we expect for the 'spatial' correlation length

$$
\xi_{\perp}^{-1}=L^{-1} S\left(C_{1} t L^{1 / \nu_{\perp}}, C_{2} h L^{(\beta+\gamma) / \nu_{\perp}}\right)
$$

with a universal scaling function $S$ and the same metric factors $C_{1,2}$ as in (21). While this analysis was phrased in terms of the transverse spin-spin correlation length $\xi_{\perp}=\xi_{\perp, \sigma}$, similar arguments should hold for the 'spatial' correlation lengths $\xi_{\perp, i}$ of any other physical observable, with $S$ in (23) being replaced by an appropriate function $S_{i}$. The scaling functions $Y$ and $S_{i}$ should depend on the boundary conditions and, if $d \geq 2$, on the shape of the finite 'spatial' domain. Note that $L$ refers here to the physical length, which cannot be equated to the number of sites $N$ times the lattice constant in non-square lattices [5,22]. For a recent example of this in the $2 D$ Ising model context, see [23].

Finally, the 'temporal' correlation lengths $\xi_{\|, i}$ should read

$$
\xi_{\|, i}^{-1}=L^{-\theta} D_{0} R_{i}\left(C_{1} t L^{1 / \nu_{\perp}}, C_{2} h L^{(\beta+\gamma) / \nu_{\perp}}\right)
$$

with universal scaling functions $R_{i}$ and again the same metric factors $C_{1,2}$ as before. The value of $D_{0}$ is related to the dimensionful constant which occurs in the energy-momentum dispersion relation of the underlying continuum field theory and cannot be found straightforwardly. However, at criticality $(t=h=0)$ ratios of 'temporal' correlation lengths $\xi_{\|, i} / \xi_{\|, j}$ should tend to universal constants in the $L \rightarrow \infty$ limit. The universality of these ratios would not have immediately been obvious from straightforward finite-size scaling.

Eq. (23) is the main result of this section. It provides the natural generalisation of the Privman-Fisher form (1) to the case of equilibrium anisotropic scaling. It is immediate to include further physical parameters into the analysis. We emphasize that (i) translation invariance and (ii) hyperscaling was required in deriving this result. We stress that we considered finite sizes in the 'spatial' direction and obtain universality for the spatial correlation lengths $\xi_{\perp, i}$ only.

\section{UNIVERSAL FINITE-SIZE AMPLITUDES OUT OF EQUILIBRIUM}

We have seen that in equilibrium systems with anisotropic scaling and in a geometry where the 'spatial' directions are of finite extent $L$ while the 'temporal' directionis infinite, the 'spatial' correlation lengths have a universal finitesize scaling amplitude. We now ask whether this result generalizes towards more general forms of dynamical scaling, 
without appealing to the special properties of equilibrium systems. Fluctuations in non-equilibrium systems can be treated in terms of dynamic functionals via Martin-Siggia-Rose theory 24] 27. To be specific, we shall work in a setting of reaction-diffusion processes, of which directed percolation is a common example, see [28 31] for reviews. We shall continue to denote time by $r_{\|}$and space by $r_{\perp}$. As before, $t$ measures the distance from the steady-state critical point and $h$ denotes an external field (e.g., for directed percolation $t=p-p_{c}$ and $h$ is the rate of a process $\emptyset \rightarrow A$ ). For the sake of technical simplicity, we shall assume translation invariance throughout. As in equilibrium, we have to trace the non-universal metric factors and this is most conveniently done in the bulk.

Physical quantities of interest are the mean particle density $\rho$, the survival probability $P$ and the pair connectedness function $G=G\left(r_{\perp}^{\prime}, r_{\|}^{\prime} ; r_{\perp}, r_{\|}\right)$, which is defined as the probability that the sites $\left(r_{\perp}^{\prime}, r_{\|}^{\prime}\right)$ and $\left(r_{\perp}, r_{\|}\right)$are connected by a direct path [29,30]. Because of translation invariance $G=G\left(r_{\perp}^{\prime}-r_{\perp}, r_{\|}^{\prime}-r_{\|}\right)$, which will be used throughout. These quantities are expected to satisfy the scaling behaviour

$$
\begin{aligned}
& \rho\left(r_{\perp}, r_{\|} ; t, h\right)=b^{-x_{\rho}} \rho\left(\frac{r_{\perp}}{b}, \frac{r_{\|}}{b^{z}} ; t b^{y_{t}}, h b^{y_{h}}\right)=D_{1 \rho} \xi_{\perp}^{-x_{\rho}} \mathcal{E}^{ \pm}\left(\frac{r_{\perp}}{\xi_{\perp}}, D_{0} \frac{r_{\|}}{\xi_{\perp}^{z}} ; D_{2} h|t|^{-y_{h} / y_{t}}\right) \\
& P\left(r_{\perp}, r_{\|} ; t, h\right)=b^{-x_{P}} P\left(\frac{r_{\perp}}{b}, \frac{r_{\|}}{b^{z}} ; t b^{y_{t}}, h b^{y_{h}}\right)=D_{1 P} \xi_{\perp}^{-x_{P}} \mathcal{F}^{ \pm}\left(\frac{r_{\perp}}{\xi_{\perp}}, D_{0} \frac{r_{\|}}{\xi_{\perp}^{z}} ; D_{2} h|t|^{-y_{h} / y_{t}}\right) \\
& G\left(r_{\perp}, r_{\|} ; t, h\right)=b^{-x_{G}} G\left(\frac{r_{\perp}}{b}, \frac{r_{\|}}{b^{z}} ; t b^{y_{t}}, h b^{y_{h}}\right)=D_{1 G} \xi_{\perp}^{-x_{G}} \mathcal{G}^{ \pm}\left(\frac{r_{\perp}}{\xi_{\perp}}, D_{0} \frac{r_{\|}}{\xi_{\perp}^{z}} ; D_{2} h|t|^{-y_{h} / y_{t}}\right)
\end{aligned}
$$

where the $x$ 's are scaling dimensions and $y_{t, h}$ renormalization group eigenvalues, the $D$ 's are non-universal metric factors, $\mathcal{E}, \mathcal{F}, \mathcal{G}$ are universal scaling functions where the index distinguishes between the cases $t>0$ and $t<0$, $\xi_{\perp}=\xi_{0}|t|^{-\nu_{\perp}}$ is the spatial, $\xi_{\|}=\xi_{\perp}^{z} / D_{0}$ is the temporal correlation length and $z$ is the dynamical exponent (as before $\left.y_{t}=1 / \nu_{\perp}\right)$.

In the steady state, and for $h=0$, one expects $\rho \sim t^{\beta}$ and $P \sim t^{\beta^{\prime}}$. In general, the two exponents $\beta$ and $\beta^{\prime}$ are distinct from each other. For spatial translation invariance, the dependence on $r_{\perp}$ drops out for both $\rho$ and $P$ and in the steady state (i.e. $r_{\|} \rightarrow \infty$ ) one has

$$
\begin{aligned}
& \rho(t, h)=D_{1 \rho} \xi_{0}^{-\beta / \nu_{\perp}} \widetilde{\mathcal{E}}^{ \pm}\left(D_{2} h|t|^{-y_{h} / y_{t}}\right)|t|^{\beta} \\
& P(t, h)=D_{1 P} \xi_{0}^{-\beta^{\prime} / \nu_{\perp}} \widetilde{\mathcal{F}}^{ \pm}\left(D_{2} h|t|^{-y_{h} / y_{t}}\right)|t|^{\beta^{\prime}}
\end{aligned}
$$

where $x_{\rho}=\beta / \nu_{\perp}, x_{P}=\beta^{\prime} / \nu_{\perp}$ and $\widetilde{\mathcal{E}}^{ \pm}=\lim _{r_{\|} \rightarrow \infty} \mathcal{E}^{ \pm}$and similarly for $\mathcal{F}$. We also consider the auto-connectedness (that is $r_{\perp}=r_{\perp}^{\prime}$ ) in the steady state

$$
G(0, \infty ; t, h)=: G(t, h)=D_{1 P} \xi_{0}^{-x_{G}} \widetilde{\mathcal{G}}^{ \pm}\left(D_{2} h|t|^{-y_{h} / y_{t}}\right)|t|^{x_{G} \nu_{\perp}}
$$

In the active phase $(t>0)$, the surviving clusters will create an average density $\sim|t|^{\beta}$ in the interior of the spreading cone. Therefore, the auto-connectedness should in the steady state saturate at the value 32.

$$
G(t, h)=\rho(t, h) P(t, h)
$$

Comparison of the scaling forms then yields, setting $h=0$,

$$
x_{G}=\left(\beta+\beta^{\prime}\right) / \nu_{\perp}, \quad D_{1 G}=D_{1 \rho} D_{1 P} \frac{\widetilde{\mathcal{E}}^{ \pm}(0) \widetilde{\mathcal{F}}^{ \pm}(0)}{\widetilde{\mathcal{G}}^{ \pm}(0)}
$$

Usually, $x_{G}=d-\theta z$ is expressed in terms of the initial critical slip exponent $\theta$ [33], which makes it apparent that the expression (29) is in fact a generalized hyperscaling relation 229,30,32].

Next, we consider the total mass $M$ of the cluster [30], given by

$$
M(t, h):=\int_{\mathbb{R}^{\mathrm{d}}} \mathrm{d}^{d} r_{\perp} \int_{0}^{\infty} \mathrm{d} r_{\|} G\left(r_{\perp}, r_{\|} ; t, h\right)=\frac{D_{1 G}}{D_{0}} \xi_{\perp}^{\gamma / \nu_{\perp}} \overline{\mathcal{G}}^{ \pm}\left(D_{2} h|t|^{-y_{h} / y_{t}}\right)
$$

where eq. 25) was used and $\overline{\mathcal{G}}^{ \pm}$is a new universal function related to $\mathcal{G}^{ \pm}$. Also

$$
\gamma=d \nu_{\perp}+\nu_{\|}-\beta-\beta^{\prime}
$$


which is the analogue of the hyperscaling relation of the equilibrium systems.

While the discussion so far has been completely general, we now appeal to two properties which are valid for systems in the directed percolation universality class, but need not be generically valid. First, we consider a directed percolation process in the presence of a weak field $h$ (physically, $h$ parametrises the rate of a particle creation process $\emptyset \rightarrow A$ ). A site at a given time becomes active if it is connected with at least one active site in the past, where a particle was created by the field. The number of such sites is equal to the cluster size, the probability to become active is given by the density [30]

$$
\rho(t, h) \simeq 1-(1-h)^{M(t, h)} \simeq h M(t, h)
$$

for $h$ small. Therefore,

$$
M(t, 0)=\left.\frac{\partial \rho(t, h)}{\partial h}\right|_{h=0}
$$

Comparison with the scaling forms for $\rho$ and $M$ leads to

$$
y_{h} / y_{t}=\beta+\gamma, D_{1 P}=D_{0} D_{2} \xi_{0}^{-(\beta+\gamma) / \nu_{\perp}} \mathcal{A}^{ \pm}
$$

where $\mathcal{A}^{ \pm}$is an universal amplitude. Second, directed percolation is special in the sense that there is a 'duality' symmetry which can be used to show that 34]

$$
\rho(t, h)=P(t, h)
$$

As a consequence, $\beta=\beta^{\prime}$ and $D_{1 \rho}=D_{1 P}$ for directed percolation and we thus have, combining eqs. (26, 30,34)

$$
\begin{gathered}
\rho(t, h)=D_{0} D_{2} \xi_{0}^{-d-z}|t|^{\beta} \hat{\mathcal{M}}_{1}^{ \pm}\left(D_{2} h|t|^{-\beta-\gamma}\right) \\
M(t, h)=D_{0} D_{2}^{2} \xi_{0}^{-d-z}|t|^{-\gamma} \hat{\mathcal{M}}_{2}^{ \pm}\left(D_{2} h|t|^{-\beta-\gamma}\right)
\end{gathered}
$$

with universal functions $\hat{\mathcal{M}}_{n}^{ \pm}(x)=\mathrm{d}^{n} \hat{\mathcal{M}}^{ \pm}(x) / \mathrm{d} x^{n}$ and where the hyperscaling relation eq. (31) has been used. We therefore recover the analogues of eqs. (15,16) found in equilibrium. Finally, we define a new function $\mu=\mu(t, h)$ by $\rho(t, h)=\partial \mu(t, h) / \partial h$, which implies

$$
\mu(t, h)=D_{0} \xi_{0}^{-d-z}|t|^{(d+z) \nu_{\perp}} \hat{\mathcal{M}}^{ \pm}\left(D_{2} h|t|^{-\beta-\gamma}\right)
$$

In particular, as we did before at equilibrium, we have because of $\xi_{\|}=\xi_{\perp}^{z} / D_{0}$ that

$$
\mu(t, 0) \xi_{\perp}^{d}(t, 0) \xi_{\|}(t, 0) \underset{t \rightarrow 0}{\rightarrow} \text { univ. constant, }
$$

which is indeed the analogue of the result (22).

At last, we consider a geometry of finite size $L$ in space but of infinite extent in time. Again, by analogy with section 2 , we postulate that in this finite geometry merely the scaling functions are modified

$$
\hat{\mathcal{M}}_{n}^{ \pm}=\hat{\mathcal{M}}_{n}^{ \pm}\left(D_{2} h|t|^{-\beta-\gamma} ; L \xi_{\perp}^{-1}\right)
$$

and without introducing any further metric factor. Indeed, we can then scale out $L$ and, because of eq. (38), arrive at the same scaling forms $(23,24)$ as had been found before for the spatial and temporal correlation lengths in anisotropic equilibrium systems, at least for systems in the directed percolation universality class. Since directed percolation is known to be equivalent to an equilibrium (in fact, purely geometrical) problem, the existence of universal finite-size amplitudes in this class is not too surprising and might have been anticipated from the discussion in the previous section.

While the spatial correlation length $\xi_{\perp}$ may not be always a very accessible quantity, its universality may also be tested by considering the spatial moment (let $t=h=0$ for simplicity)

$$
\begin{aligned}
R_{L}^{(n)}:=\left\langle r_{\perp}^{n}\right\rangle & =\frac{\int_{\Lambda(L)} \mathrm{d}^{d} r_{\perp} \int_{0}^{\infty} \mathrm{d} r_{\|} r_{\perp}^{n} G\left(r_{\perp}, r_{\|} ; L / \xi_{\perp}\right)}{\int_{\Lambda(L)} \mathrm{d}^{d} r_{\perp} \int_{0}^{\infty} \mathrm{d} r_{\|} G\left(r_{\perp}, r_{\|} ; L / \xi_{\perp}\right)} \\
& =\xi_{\perp}^{n} \frac{\int_{\Lambda\left(L / \xi_{\perp}\right)} \mathrm{d}^{d} r_{\perp} \int_{0}^{\infty} \mathrm{d} r_{\|} r_{\perp}^{n-x_{G}} \mathcal{G}^{ \pm}\left(r_{\perp}, r_{\|} ; L / \xi_{\perp}\right)}{\int_{\Lambda\left(L / \xi_{\perp}\right)} \mathrm{d}^{d} r_{\perp} \int_{0}^{\infty} \mathrm{d} r_{\|} r_{\perp}^{-x_{G}} \mathcal{G}^{ \pm}\left(r_{\perp}, r_{\|} ; L / \xi_{\perp}\right)} \\
& =\xi_{\perp}^{n} \Xi_{n}\left(L / \xi_{\perp}\right) \\
& =L^{n} \widetilde{\Xi}_{n}\left(L / \xi_{\perp}\right)
\end{aligned}
$$


where $\Lambda(L)$ is a $d$-dimensional hypercube of linear extent $L$ and $\Xi_{n}$ and $\widetilde{\Xi}_{n}$ are universal functions. Since there is no metric factor in the argument of $\widetilde{\Xi}_{n}$, the universality of the finite-size scaling amplitude of $\xi_{\perp}$ is equivalent to the universality of the finite-size scaling amplitude of $R_{L}^{(n)}$ (on the other hand, the temporal moment $\left\langle r_{\|}^{n}\right\rangle \sim\left(D_{0}^{-1} L^{z}\right)^{n}$ has a non-universal amplitude). The universality of these moments is a somewhat stronger statement than the universality of certain ratios of moments $\left\langle\rho^{n}\right\rangle$ of the particle density $\rho$ which has recently been verified in $1 D$ and in $2 D$ for several models in the directed percolation universality class [35].

In summary, we have seen that for systems in the directed percolation universality class, the special properties eqs. (33, 35), taken together with the general relation (28), are sufficent to rederive the universal finite-size scaling form (23) of the spatial correlation length, in spite of the absence of the fluctuation-dissipation relation. It is not yet clear wether there exist more general arguments which would permit us to arrive at the same result without appealing to either (33) or (35). However, we shall in the next section present numerical evidence that the universal finite-size scaling forms (23) for $\xi_{\perp}$ or (24) for $\xi_{\|}$might be more generally valid.

\section{REACTION-DIFFUSION PROCESSES}

The new information contained in (23) which goes beyond the standard RG ideas is the universality of the finite-size scaling amplitude $L \xi_{\perp}^{-1}$ precisely at criticality (and similarly the universality of all ratios $\xi_{\|, i} / \xi_{\|, j}$ ). Since for the time being, the derivation of this universality for non-equilibrium systems appears to be restricted to directed percolation, we use the pair contact process and the annihilation-coagulation model to test the universality hypothesis advanced in sections 2 and 3 quantitatively.

The pair contact process [36] has been intensively studied recently. It is a reaction-diffusion system, where particles move and react on a lattice. While each lattice site can be either empty or be occupied by a single particle, the following microscopic moves are permitted

$$
\begin{aligned}
& \left\{\begin{array}{l}
A A \emptyset \rightarrow A A A \\
\emptyset A A \rightarrow A A A
\end{array} \text { with rate } \frac{(1-p)(1-d)}{2}\right. \\
& A A \rightarrow \emptyset \emptyset \quad \text { with rate } p(1-d) \\
& A \emptyset \leftrightarrow \emptyset A \quad \text { with rate } d
\end{aligned}
$$

and are parametrized by the diffusion constant $d$ and the pair annihilation rate $p$.

While in the case without diffusion $(d=0)$, the steady-state transition between the active and the absorbing state was found to be in the directed percolation universality class [36, 35, 37], the effects of adding diffusion were first studied using field-theoretical methods, considering a bosonic field theory without any restriction on the number of particles per site (which leads to a divergent particle density in the active phase) [38]. It was shown that the entire absorbing phase is critical and in the universality class of diffusion-annihilation (see below). Because of the nonrenormalizability of the underlying field theory, no quantitative information about the transition towards the active state could be obtained. The first quantitative informations were obtained 39 through the use of density matrix renormalization group (DMRG) techniques 40 42. The steady state phase diagram is shown in figure 1 and there is a general agreement between DMRG and Monte Carlo studies on the location of the critical point $p_{c}(d)$ [39,43, 46 .

The annihilation-coagulation model is formulated in the same way, with the allowed reactions

$$
\begin{array}{ll}
A A \rightarrow A \emptyset, \emptyset A & \text { with rate } d \gamma \\
A A \rightarrow \emptyset \emptyset & \text { with rate } 2 d \alpha \\
A \emptyset \leftrightarrow \emptyset A & \text { with rate } d
\end{array}
$$

parametrized by $\alpha$ and $\gamma$ for annihilation and coagulation, respectively. The long-time behaviour is the model is always algebraic, i.e. the mean particle density $\rho(t) \sim t^{-1 / 2}$ in $1 D$, see [28 31, 47] and references therein. 


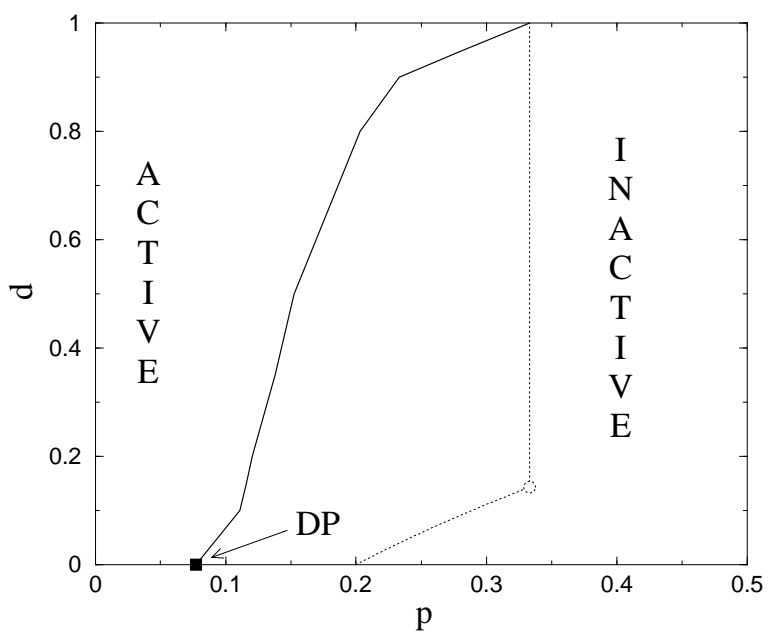

FIG. 1. Steady state phase diagram of the pair contact process. The dotted lines are the phase boundaries according to pair mean field theory, while the full line gives the active-inactive transition in $1 D$. DP marks the steady-state transition in the directed percolation universality class at $d=0$.

In this paper, we shall use the Hamiltonian formulation of reaction-diffusion processes [48 52], which starts from the master equation

$$
\frac{\partial|P(t)\rangle}{\partial t}=-H|P(t)\rangle
$$

where $|P(t)\rangle$ a state vector and $H$ is referred to as "quantum" Hamiltonian (for recent reviews, see [30,47]). For a chain with $L$ sites, $H$ is a stochastic $2^{L} \times 2^{L}$ matrix with elements

$$
\langle\sigma|H| \tau\rangle=-w(\tau \rightarrow \sigma),\langle\sigma|H| \sigma\rangle=\sum_{\tau \neq \sigma} w(\sigma \rightarrow \tau)
$$

where $|\sigma\rangle,|\tau\rangle$ are the state vectors of the particle configurations $\sigma, \tau$ and $w$ are the transition rates. It is well-known that the ground state energy of the pair contact process $E_{0}=E_{1}=0$ is twofold degenerate for $d \neq 0$ [39]. The energy gap $\Gamma=E_{2}-E_{0}$, calculated from the second excited state, is the inverse relaxation time or, in the notation of section 2, the inverse 'temporal' correlation length $\xi_{\|}^{-1}=\Gamma$ towards the steady state. We shall consider both free and periodic boundary conditions.

1. First, we discuss the pair contact process (41). We find the following, surprisingly simple, finite-size scaling behaviour for the gap $\Gamma_{L}$ in the entire absorbing phase, that is, for all $p \geq p_{c}(d)$, namely

$$
\Gamma_{L}=a d L^{-2}\left(1+O\left(L^{-1}\right)\right)
$$

where $a$ depends on the boundary condition

$$
a= \begin{cases}2 \pi^{2} & ; \text { periodic } \\ \pi^{2} & ; \text { free }\end{cases}
$$

but is independent of both $p$ and $d$.

Before deriving (46), we argue that this result confirms the universality of the correlation length amplitudes discussed in section 3. Indeed, for systems in the diffusion-annihilation universality class already the inverse 'temporal' correlation length $\Gamma$ has a universal amplitude, provided that the value of the diffusion constant $d$ is fixed. The universality of the reaction-diffusion process $2 A \rightarrow \emptyset$ has been discussed using field theory methods [53 55]. From the renormalisation group equations, it can be shown that the value of the diffusion constant $d$ is not renormalised through the effects of the interaction of the particles and simply stays at its bare value. The bare value of $d$ is the value it has in the original lattice formulation of the problem. Since the diffusion constant sets the time scale, we expect for the gap $\Gamma \sim L^{-2} d$. These calculations [53 55] apply to the process $2 A \rightarrow \emptyset$ which corresponds to the case $p=1$ in the model at hand. However, it is known that in the entire absorbing phase, the extra interactions coming from the reaction $2 A \rightarrow 3 A$ are irrelevant [38]. Therefore, they should not modify the value of $d$ (since we 
only consider here the inactive phase, we leave aside the question how $d$ evolves under renormalization in the active phase). Consequently, the proportionality of $\Gamma$ and $d$ in eq. (45) comes from the non-renormalisation of $d$. That non-renormalisation is a special property of the diffusion-annihilation universality class. That is consistent with the scaling form (24) for $\xi_{\|}$and we can identify $D_{0}=d$. Finally, the $p$-independence of the amplitude $a$ in eq. (46) is an example of the universality of the finite-size scaling amplitude $R(0,0)$ in eq. (24), which in turn implies the universality of $S(0,0)$ in eq. (23).

We now derive eqs. (45, 46). We need the lowest non-vanishing eigenvalue $\Gamma=E_{2}$ of the quantum Hamiltonian $H$. For $p=1$, it turns out that the spectrum of $H$ is equal to the spectrum of an XXZ Heisenberg quantum chain $H_{\mathrm{XXZ}}$. The lowest gap of $H_{\mathrm{Xxz}}$ can be found from the coordinate Bethe ansatz [56,57). This reproduces eq. (46) for $p=1$ and all values of $d$. The details of the calculation are presented in the appendix.

We point out, however, that for $d=1 / 2$ and free boundary conditions only, the Bethe ansatz equations have a closed-form solution. The exact lowest gap for any finite number of sites $L$ is

$$
\Gamma_{L}=1-\cos \frac{\pi}{L+1} ; d=\frac{1}{2}, p=1, \text { free b.c. }
$$

in agreement with $(45,46)$. This had been conjectured before on the basis of numerical data [42] (closed-form solutions for slightly different $\Delta=1 / 2 \mathrm{XXZ}$ chains have been discussed recently in [58]).

For $p \neq 1$, the PCPD is not related to any known integrable model and we revert to numerical methods. We consider the normalized amplitudes

$$
A_{L}^{(\mathrm{P})}=L^{2} \Gamma_{L} /\left(2 \pi^{2} d\right), A_{L}^{(\mathrm{F})}=L^{2} \Gamma_{L} /\left(\pi^{2} d\right)
$$

defined for periodic $(\mathrm{P})$ and free $(\mathrm{F})$ boundary conditions, respectively. If and only if (46) is correct, the amplitudes $A_{L}^{(\mathrm{P})}$ and $A_{L}^{(\mathrm{F})}$ should converge towards unity in the $L \rightarrow \infty$ limit.

In table 1 we show data for periodic boundary conditions. These were obtained from diagonalizing $H$ through the standard Arnoldi algorithm [3]. Translation and parity invariance were used to blockdiagonalize $H$, and lead to matrices of size $\approx 2^{L} /(2 L)$ for $L$ sites. In table II data for free boundary conditions are shown. They were obtained by applying the density matrix renormalization group (DMRG) method 40 42] to the pair contact process [39].

Clearly, the data for both $A_{L}^{(\mathrm{P})}$ and $A_{L}^{(\mathrm{F})}$ at finite values of $L$ are in general quite far away from unity. We also see that the raw data tend to be closer to unity for smaller values of $d$. However, since the systematic variation of these amplitudes with $L$ is huge, a precise $L \rightarrow \infty$ extrapolation must be performed. We have used the BST extrapolation algorithm [59] which has established itself as a reliable and precise method for the extrapolation of finite-lattice sequences arising in both equilibrium and non-equilibrium critical phenomena. The parameter $\omega$ describes the (effective) leading finite-size correction of a sequence $A_{L}=A_{\infty}+\mathcal{A}_{1} L^{-\omega}+\ldots$ and must be chosen to optimize convergence [3,59].

In all cases, we find that the extrapolated amplitudes

$$
A_{\infty}^{(\mathrm{P})}:=\lim _{L \rightarrow \infty} A_{L}^{(\mathrm{P})} \simeq 1, A_{\infty}^{(\mathrm{F})}:=\lim _{L \rightarrow \infty} A_{L}^{(\mathrm{F})} \simeq 1
$$

within the numerical accuracy of the extrapolation and in full agreement with eq. (46). The need for $L \rightarrow \infty$ extrapolation also means that the universal infinite-size amplitudes may be hard to see in, say, Monte Carlo simulations. We also give the effective value of $\omega$ for each sequence. In view of the exact result $\omega=1$ for $p=1$, see (57,62), it is satisfying that $\omega$ stays close to one.

In general, for a given $d$ convergence is best for $p$ close to 1 and decreases when $p$ is lowered. The lowest values of $p$ given in table 1 are more or less the smallest ones for which a reliable convergence of the amplitudes could still be observed. In varying $d$, we see that data converge best for relatively small $d$ and that close to $d=1$ the cross-over towards mean-field behaviour [39] affects the finite-size scaling of the amplitudes. Comparing the data for periodic and free boundary conditions for the same values of $p$ and $d$, we observe that the $A_{\infty}^{(\mathrm{F})}$ are closer to unity than the $A_{\infty}^{(\mathrm{P})}$ for the same value of $L$ (and in contrast to the usual expectation that finite-size corrections should be smallest for periodic boundary conditions). For $p=1$, these remarks are confirmed analytically, see eqs. (57,62).

All in all, the extrapolated amplitudes converge over a large range of values of $p$ and $d$ towards unity, in agreement with (46). Therefore, the PCPD in the inactive phase confirms the universality of the correlation length finite-size scaling amplitude as derived in section 2. Since the entire inactive phase is expected to be in the same universality class, that result should apply even to those portions of the inactive phase where our relatively short chains did not permit us to carry out a precise extrapolation. 
2. Second, we briefly discuss the annihilation-coagulation model (42). It is well known [53,60 63 that the quantum Hamiltonian $H=H(\alpha, \gamma)$ is similar to the quantum Hamiltonian $H(\alpha+\gamma, 0)$ of pure annihilation. Therefore eqs. (45,46) also apply to this model, independently of $\alpha$ and $\gamma$. If we take $\alpha+\gamma=1$, the steady-state particle density amplitude $\lim _{L \rightarrow \infty} L \rho(L)=(1+\alpha / \gamma) /(1+2 \alpha / \gamma)$ depends on the branching ratio $\alpha / \gamma$ and is not universal.

Eqs. (45,46) state that for the finite-size amplitude of $\Gamma$ is independent of the ratio $r=\alpha+\gamma$ of the reaction rate and the diffusion rate $d$. In the light of the universality hypothesis of sections 2 and 3 , the observed $r$-independence of the amplitude $L^{2} \Gamma$ means that the critical exponents of the pair annihilation process $2 A \rightarrow \emptyset$ (or the equivalent coagulation process $2 A \rightarrow A\left[53[60]\right.$ ) should also be independent of $r$, i.e. the mean particle density $\rho(t) \sim t^{-\delta}$ with $\delta=1 / 2$. While that had been anticipated long ago by many people, exact lattice calculations only exist for $r=1$, see [30,47. The only published verifications of the $r$-independence of $\delta$ we are aware of either used purely numerical methods [62,64,65], a real-space renormalisation group scheme [66] or other ad hoc approximations [67].

The steady-state particle density should scale as

$$
\rho_{L}=\left.D_{0} C_{2} L^{-\beta / \nu_{\perp}} Y^{\prime}\left(0, C_{2} h L^{d+\theta-\beta / \nu_{\perp}}\right)\right|_{h=0}
$$

(borrowing a notation from section 2, where the prime indicates the derivative with respect to the second argument and $h$ parametrises a source of particles). That is standard finite-size scaling without any readily identifiable universal amplitude and is therefore weaker than the forms of sections 2 and 3. It is known from field theory that $C_{2}$ is independent of $r$ [54] while it does depend on the branching ratio $\alpha / \gamma$ [63] which is compatible with our results.

3. Having checked the scaling function universality in some examples with known behaviour, we now illustrate how this universality might be used as a diagnostic tool. Appealing to the experience gathered in equilibrium systems [4, universal amplitudes might be expected to vary considerably more between distinct universality classes than critical exponents. Therefore, even an approximate determination of universal amplitude may allow to conclude on the universality class of the model at hand. Reconsider the phase diagram of the pair contact process in fig. 1. Presently, there is no consensus on how many universality classes should be realised along the transition line between the active and inactive phases for $d \neq 0,1$. As a starting point, we might consider pair mean field theory, which predicts two distinct universality classes along the two segments of the pair mean field transition curve [39]. The calculation of both steady-state and time-dependent critical exponents in $1 D$ from simulations 46 appear to be in agreement with this prediction. On the other hand, DMRG studies [39] and different simulations in $1 D$ [44,45, 68] only find evidence for a single universality class along the transition line.

Here, we consider the ratio $R=E_{3} / E_{2}$ of the two lowest non-vanishing eigenvalues of $H$ for free boundary conditions as obtained from the DMRG. We use the Monte Carlo estimates for $p_{c}(d)$ obtained by Grassberger 445]. $R$ is equal to the ratio of two distinct relaxation times and from eq. (24), we expect $R$ to be constant within a given universality class. Our numerical results are shown in fig. 2 .

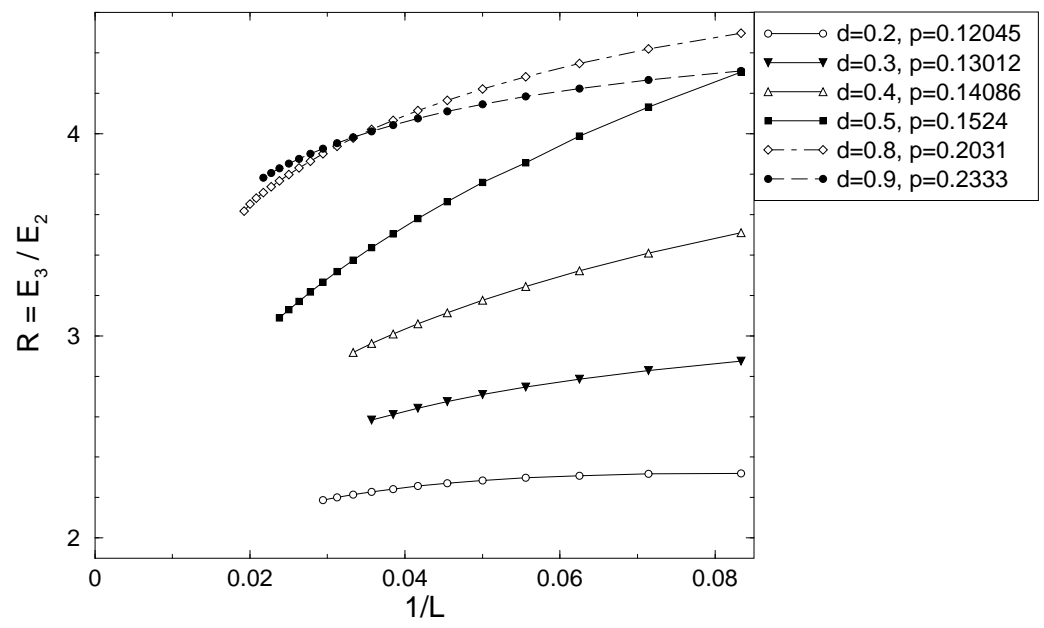

FIG. 2. Ratio $R=E_{3} / E_{2}$ of the two lowest eigenvalues of $H$ at the critical line $p=p_{c}(d)$ of the pair contact process, for free boundary conditions and several values of $d$.

At first sight, it might be possible to separate the values of $R$ into two classes, one for smaller values of $d$ (up to $d \approx 0.4-0.5$ ) and a limit $R_{\infty} \sim 2$, and one for larger values of $d$ (above $d \approx 0.8$ ) with a limit $R_{\infty} \sim 3-4$. The fact 
that $R_{\infty}$ is quite independent of $d$ for $0.2 \leq d \leq 0.4$ confirms the expected universality $\left(R_{\infty}=1\right.$ in the inactive phase because there all levels are two-fold degenerate, see appendix).

However, closer inspection reveals that already for $d=0.5$, the data for $R_{L}$ for $L$ small start out close to 4 and then begin to cross over to values close to 2 . That signals the presence of strong transient effects in this model. In addition, for the values of $L$ for which data exist, the values of $R$ for both $d=0.8$ and $d=0.9$ are close together and quite close to 4 . However, these data, in particular for $d=0.8$, also show indications that they might also cross over to smaller values of $R$ if $L$ could be increased further. The lattice sizes available are not large enough to be able to distinguish clearly between the possibilities of a single [39, 44, 45, 68] or two [46] transitions, although the possibility of a single transition appears more likely. If there is a change of the universality class along the critical line at all, fig. 2 suggests that it certainly should occur for $d>0.5$.

\section{CONCLUSIONS}

In this paper, we have tried to generalize Privman-Fisher [5] universality to (steady-state) phase transitions with dynamical scaling. For equilibrium systems the standard arguments carry over, the main ingredients being translation invariance and hyperscaling. With respect to more standard renormalisation group arguments, only the absence of non-universal metric factors in front of the scaling function for $\xi_{\perp, i}$ in eq. (23) is new. Equivalently, this may be stated, see eq. (24), as the universality of all ratios $\xi_{\|, i} / \xi_{\|, j}$.

Out of equilibrium, new arguments must be sought. In the special case of directed percolation, the peculiar properties eqs. (33, 35) were seen to be sufficient for amplitude universality. These properties may or may not be available in other universality classes, but we found some numerical evidence in several reaction-diffusion systems that the universal finite-size scaling forms (23,24) might indeed hold in general. In the annihilation-coagulation model and the pair contact process (inactive phase), we found that the finite-size scaling amplitude of the leading relaxation time is indeed independent of the irrelevant parameters we considered. Further evidence in favour of universality was found by studying the active-inactive transition line in the pair contact process. In addition, our data for the ratio of the two leading relaxation times appear to favour a single universality class along that transition line for $0<d<1$. It remains an open question how to derive the universal scaling forms (23,24) in general non-equilibrium systems.

Recently, the reaction-diffusion process $2 A \rightarrow A$ and $A \emptyset A \rightarrow 3 A$ has been studied [69]. If the coagulation rate is equal to the diffusion rate, the model is exactly solvable. It remains in the universality class of diffusion-annihilation for all values of the particle production rate $\lambda$. For periodic boundary conditions, the exact amplitude of the leading inverse relaxation time is $\lim _{L \rightarrow \infty} L^{2} \Gamma_{L}=2 \pi^{2} d$, independently of $\lambda$, as to be expected from (45,46).

Finally, upon identification the universal finite-size scaling amplitude of the transverse 'spatial' correlation lengths $\xi_{\perp, i}$, it might be tempting to ask if, in analogy to equilibrium [6 10], there could be relations of universal finite-size scaling amplitudes with some exponents. To answer this question would require a set of worked-out examples on which some hypothesis of this kind could be tried out.

\section{ACKNOWLEDGMENTS}

We thank E. Carlon for his contributions in developing the application of the DMRG method to non-equilibrium systems, P. Grassberger for kindly sharing his data on $p_{c}(d)$ before publication, H. Hinrichsen for discussions and L. Turban for a critical reading of the manuscript. MH thanks the Complexo Interdisciplinar of the University of Lisbon for warm hospitality. This work was supported by the French-German Procope programme.

\section{APPENDIX: BETHE ANSATZ CALCULATION}

We calculate the lowest gap $\Gamma=E_{2}$ for $p=1$ in the pair contact process and derive the amplitude $a$ in (46).

In the $p=1$ case, only the pair annihilation $A A \rightarrow \emptyset \emptyset$ survives. It is well known [52] that in this case, the quantum Hamiltonian can be decomposed $H=H_{0}+H_{1}$ in such a way that the eigenvalue spectrum of $H$ is independent of $H_{1}$, viz. spec $(H)=\operatorname{spec}\left(H_{0}\right)$. The latter is related to the spectrum of the XXZ Heisenberg chain which can be found from the coordinate Bethe ansatz [56,57].

We begin with the periodic case. The spectrum-generating part of $H$ is

$$
H_{0}=d H_{\mathrm{XXZ}}(\Delta, t)+\frac{1}{4}(1+d) L
$$


where

$$
H_{\mathrm{XXZ}}(\Delta, t)=-\frac{1}{2} \sum_{i=1}^{L}\left[\sigma_{i}^{x} \sigma_{i+1}^{x}+\sigma_{i}^{y} \sigma_{i+1}^{y}+\Delta \sigma_{i}^{z} \sigma_{i+1}^{z}+t \sigma_{i}^{z}\right]
$$

and

$$
\Delta=\frac{3 d-1}{2 d}, t=\frac{1-d}{d}
$$

Since the total spin $S^{z}:=\sum_{i=1}^{L} \sigma_{i}^{z}$, commutes with $H_{\mathrm{XXZ}}$, the eigenstates $H_{0}\left|\psi_{n}\right\rangle=E_{n}\left|\psi_{n}\right\rangle$ can be classified in terms of the number $n$ of reversed spins, viz. $S^{z}\left|\psi_{n}\right\rangle=(L-2 n)\left|\psi_{n}\right\rangle$. The lowest states with $n=0,1$ have zero energy and correspond to the two steady states of the model. The lowest gap $\Gamma$ is found in the sector $n=2$. From [57], one has

$$
E_{2}=2 d\left(2-\cos k-\cos k^{\prime}\right), \quad L k=2 \pi I-\Theta\left(k, k^{\prime}\right), \quad L k^{\prime}=2 \pi I^{\prime}-\Theta\left(k^{\prime}, k\right)
$$

where

$$
\Theta\left(k, k^{\prime}\right)=2 \arctan \frac{\Delta \sin \left(\left(k-k^{\prime}\right) / 2\right)}{\cos \left(\left(k+k^{\prime}\right) / 2\right)-\Delta \cos \left(\left(k-k^{\prime}\right) / 2\right)}
$$

and $I, I^{\prime}= \pm \frac{1}{2}, \pm \frac{3}{2}, \ldots$ are distinct half-integers. The total momentum of the corresponding state is $P=k+k^{\prime}=$ $2 \pi\left(I+I^{\prime}\right) / L^{\prime}$. The lowest energy gap will have $P=0$, or $k^{\prime}=-k$. Furthermore, the lowest energy state corresponds to the choice $I=-I^{\prime}=1 / 2$, as can be checked by considering the special case $\Delta=0$ (because of the symmetry between $k$ and $k^{\prime}$, all levels are two-fold degenerate). We find

$$
\Gamma=4 d(1-\cos k), \tan \frac{L k-\pi}{2}=-\frac{\Delta \sin k}{1-\Delta \cos k}
$$

For $L$ large, the solution of the second equation (56) is

$$
k \simeq \frac{\pi}{L}\left(1-\frac{2 \Delta}{1-\Delta} \frac{1}{L}+\ldots\right)
$$

Inserting this into (56), we arrive indeed at the first case of eqs. (45,46).

Second, we consider free boundary conditions. The spectrum-generating part of $H$ is

$$
\begin{gathered}
H_{0}=d H_{\mathrm{XXZ}}(\Delta, t, r)+\frac{1}{4}(1+d)(L-1) \\
H_{\mathrm{XXZ}}(\Delta, t, r)=-\frac{1}{2}\left\{\sum_{i=1}^{L-1}\left[\sigma_{i}^{x} \sigma_{i+1}^{x}+\sigma_{i}^{y} \sigma_{i+1}^{y}+\Delta \sigma_{i}^{z} \sigma_{i+1}^{z}\right]+r\left(\sigma_{1}^{z}+\sigma_{L}^{z}\right)+t \sum_{i=1}^{L} \sigma_{i}^{z}\right\}
\end{gathered}
$$

where we used (53) and $r=-t / 2$. Again, $\Gamma$ will be the lowest energy in the $n=2$ sector. From the Bethe ansatz [56], one has

$$
E_{2}=2 d\left(2-\cos k-\cos k^{\prime}\right), e^{2 \mathrm{i}(L-1) k}=\left(\frac{f_{-k}(r, \Delta)}{f_{k}(r, \Delta)}\right)^{2} e^{-\mathrm{i} \Theta\left(k, k^{\prime}\right)+\mathrm{i} \Theta\left(-k, k^{\prime}\right)}
$$

where $f_{k}(a, b)=a-b+e^{\mathrm{i} k}$. A similar equation holds for $k^{\prime}$, where $k$ and $k^{\prime}$ are exchanged with respect to (60). The lowest excitations in the $n=2$ sector are found for $k^{\prime}=0$ (up to a two-fold degeneracy). Since $r-\Delta=-1$, using (55) and taking the logarithm, we find

$$
\Gamma=2 d(1-\cos k), \quad L k=\pi(I+1)-2 \arctan \left(\frac{\Delta}{1-\Delta} \tan \frac{k}{2}\right)
$$

where $I=0,1,2,3, \ldots$. The lowest gap is obtained for $I=0$, as can be checked for $\Delta=0$. In analogy to the periodic case, we rewrite the second equation in (61) in the form $\tan ((L k-\pi) / 2)=-\Delta /(1-\Delta) \tan (k / 2)$. For $L$ large, the solution is 


$$
k \simeq \frac{\pi}{L}\left(1-\frac{\Delta}{1-\Delta} \frac{1}{L}+\ldots\right)
$$

from which the second case in eqs. (45,46) follows.

Evidently, for $\Delta=0$, that is $d=1 / 3$, we recover for both boundary conditions the well-known results found from free-fermion methods. A second closed solution exists for $d=1 / 2$ and free boundary conditions. Then $\Delta=1 / 2$ and the second of eqs. (61) reduces to $(L+1) k=\pi(I+1)$. The lowest gap is obtained for $I=0$ and we arrive at 47 ). We also remark that for the $\Delta=1 / 2$ XXZ chain with boundary terms such that $U_{q}(\operatorname{sl}(2))$ invariance holds, the Bethe ansatz equation can be solved analytically 58 .

* Unité Mixte de Recherche CNRS No 7556.

[1] M.E. Fisher, in F.J.W. Hahne (ed) Critical Phenomena, Springer (Heidelberg 1983), p. 1

[2] J.L. Cardy, Scaling and Renormalization in Statistical Physics, Cambridge University Press (Cambridge 1996)

[3] M. Henkel, Conformal Invariance and Critical Phenomena, Springer (Heidelberg 1999)

[4] V. Privman, P.C. Hohenberg and A. Aharony, in C. Domb and J.L. Lebowitz (eds) Phase Transitions and Critical Phenomena, Vol. 14, Academic Press (New York 1991)

[5] V. Privman and M.E. Fisher, Phys. Rev. B30, 322 (1984).

[6] J.L. Cardy, J. Phys. A: Math. Gen. 17, L385 (1984).

[7] M. Henkel, J. Phys. A: Math. Gen. 20, L769 (1987).

[8] M. Weigel and W. Janke, Phys. Rev. Lett. 82, 2318 (1999).

[9] J.L. Cardy, J. Phys. A: Math. Gen. 18, L757 (1985).

[10] M. Weigel and W. Janke, Europhys. Lett. 51, 578 (2000).

[11] R.M. Hornreich, M. Luban and M. Shtrikman, Phys. Rev. Lett. 35, 1678 (1975)

[12] J.v. Boehm and P. Bak, Phys. Rev. Lett. 42, 122 (1979).

[13] W. Selke, Phys. Rep. 170, 213 (1988).

[14] J.M. Yeomans, Solid State Physics 41, 151 (1988).

[15] W. Selke, in C. Domb and J.L. Lebowitz (eds) Phase Transitions and Critical Phenomena, Vol. 15, Academic Press (New York 1992).

[16] B. Neubert, M. Pleimling, and R. Siems, Ferroelectrics 208-209, 141 (1998).

[17] J.A. Hertz, Phys. Rev. 14, 1165 (1976).

[18] S. Sachdev, Quantum Phase Transitions, Cambridge University Press (Cambridge 1999)

[19] K. Binder and J.-S. Wang, J. Stat. Phys. 55, 87 (1989).

[20] K.-t. Leung, Phys. Rev. Lett. 66, 453 (1991).

[21] H.W. Diehl and M. Shpot, Phys. Rev. B62, 12338 (2000).

[22] S. Singh and R.K. Pathria, Phys. Rev. B36, 3769 (1987).

[23] N.Sh. Izmailian and C.-K. Hu, cond-mat/0009102.

[24] P.C. Martin, E.D. Siggia and H.H. Rose, Phys. Rev. A8, 423 (1973).

[25] R. Bausch, H.K. Janssen and H. Wagner, Z. Phys. B24, 113 (1976).

[26] C. de Dominicis, J. Physique Colloque 37, C247 (1976).

[27] C. de Dominicis and L. Peliti, Phys. Rev. B18, 353 (1978)

[28] B. Schmittmann and R.K.P. Zia, in C. Domb and J.L. Lebowitz (eds) Phase Transitions and Critical Phenomena, Vol. 17, Academic Press (New York 1995).

[29] J. Marro and R. Dickman, Nonequilibrium phase transitions in lattice models, Cambridge University Press (Cambridge 1999).

[30] H. Hinrichsen, Adv. Phys. 49, 815 (2000).

[31] D.C. Mattis and M.L. Glasser, Rev. Mod. Phys. 70, 979 (1998).

[32] J.F.F. Mendes, R. Dickman, M. Henkel and M.C. Marques, J. Phys. A: Math. Gen 27, 3019 (1994).

[33] H.K. Janssen, B. Schaub and B. Schmittmann, Z. Phys. B73, 539 (1989).

[34] P. Grassberger and A. de la Torre, Ann. of Phys. 122, 373 (1979).

[35] R. Dickman and J. Kamphorst Leal da Silva, Phys. Rev. E58, 4266 (1998).

[36] I. Jensen, Phys. Rev. Lett. 70, 1465 (1993).

[37] J. Kamphorst Leal da Silva and R. Dickman, cond-mat/9904161

[38] M.J. Howard and U.C. Täuber, J. Phys. A: Math. Gen. 30, 7721 (1997).

[39] E. Carlon, M. Henkel and U. Schollwöck, Phys. Rev. E63, 036101 (2001).

[40] S.R. White, Phys. Rev. Lett. 69, 2863 (1992); Phys. Rev. B48, 10345 (1993). 
[41] I. Peschel, X. Wang, M. Kaulke and K. Hallberg (Eds), Density-Matrix Renormalization, Springer (Heidelberg 1999)

[42] E. Carlon, M. Henkel and U. Schollwöck, Eur. Phys. J. B12, 99 (1999).

[43] J.F.F. Mendes, private communication (1999)

[44] H. Hinrichsen, Phys. Rev. E63, 036102 (2001).

[45] P. Grassberger, private communication (2000)

[46] G. Ódor, Phys. Rev. E62, R3027 (2000).

[47] G.M. Schütz, in C. Domb and J.L. Lebowitz (eds) Phase Transitions and Critical Phenomena, Vol.19, Academic Press (New York 2000)

[48] L.P. Kadanoff and J. Swift, Phys. Rev. 165, 310 (1968).

[49] M. Doi, J. Phys. A: Math. Gen. 9, 1465 and 1479 (1976).

[50] P. Grassberger and M. Scheunert, Fortschr. Phys. 28, 547 (1980).

[51] L. Peliti, J. Physique 46, 1469 (1985).

[52] F.C. Alcaraz, M. Droz, M. Henkel and V. Rittenberg, Ann. of Phys. 230, 250 (1994)

[53] L. Peliti, J. Phys A: Math. Gen 19, L365 (1986).

[54] M. Droz and L. Sasvári, Phys. Rev. E48, R2343 (1993).

[55] B.P. Lee, J. Phys. A: Math. Gen. 27, 2633 (1994).

[56] F.C. Alcaraz, M.N. Barber, M.T. Batchelor, R.J. Baxter and G.R.W. Quispel, J. Phys. A: Math. Gen. 20, 6397 (1987).

[57] F.C. Alcaraz, M.N. Barber and M.T. Batchelor, Ann. of Phys. 182, 280 (1988).

[58] V. Fridkin, Yu. Stroganov and D. Zagier, J. Phys. A: Math. Gen. 33, L121 (2000).

[59] R. Bulirsch and J. Stoer, Numer. Math. 6, 413 (1964);

M. Henkel and G. Schütz, J. Phys. A: Math. Gen. 21, 2617 (1988).

[60] K. Krebs, M.P. Pfannmüller, B. Wehefritz and H. Hinrichsen, J. Stat. Phys. 78, 1429 (1995).

[61] M. Henkel, E. Orlandini and G.M. Schütz, J. Phys. A: Math. Gen. 28, 6335 (1995)

[62] H. Simon, J. Phys. A: Math. Gen. 28, 6585 (1995).

[63] D. Balboni, P.-A. Rey and M. Droz, Phys. Rev. E52, 6220 (1995).

[64] K. Krebs, M.P. Pfannmüller, H. Simon and B. Wehefritz, J. Stat. Phys. 78, 1471 (1995).

[65] M.D. Grynberg, Phys. Rev. E57, 74 (1998).

[66] J. Hooyberghs and C. Vanderzande, J. Phys. A: Math. Gen. 33, 907 (2000).

[67] P.-A. Bares and M. Mobilia, Phys. Rev. Lett. 83, 5214 (1999) and 85, 893 (2000);

S.-C.Park, J.-M. Park and D. Kim, Phys. Rev. Lett. 85, 892 (2000).

[68] H. Hinrichsen, Physica A291, 275 (2001).

[69] M. Henkel and H. Hinrichsen, J. Phys. A: Math. Gen. 34, 1561 (2001). 
TABLE I. Estimates for the normalized amplitude $A_{L}^{(\mathrm{P})}=L^{2} \Gamma_{L} /\left(2 \pi^{2} d\right)$ for several values of $p$ and $d$ and periodic boundary conditions. The line labeled $\infty$ gives the $L \rightarrow \infty$ extrapolations obtained from the BST extrapolation algorithm [59] and $\omega$ is the effective correction exponent used in these extrapolations. In some cases the sequences are not monotonous, this is indicated by a ${ }^{*}$ at the value of $\omega$ used.

\begin{tabular}{|c|c|c|c|c|c|}
\hline & & \multicolumn{4}{|c|}{$p$} \\
\hline \multirow[t]{11}{*}{$d=0.10$} & $L$ & 0.16 & 0.25 & 0.50 & 0.75 \\
\hline & 6 & 0.7274475706 & 1.1014160997 & 1.5543363873 & 1.7166144988 \\
\hline & 8 & 0.6791992625 & 0.9951704118 & 1.3575537598 & 1.4819774944 \\
\hline & 10 & 0.6559859469 & 0.9505431819 & 1.2648992305 & 1.3631372237 \\
\hline & 12 & 0.6480035923 & 0.9318542215 & 1.2110759662 & 1.2913069885 \\
\hline & 14 & 0.6483265043 & 0.9247019281 & 1.1757710267 & 1.2431938517 \\
\hline & 16 & 0.6530990879 & 0.9229800785 & 1.1507547386 & 1.2087157132 \\
\hline & 18 & 0.6602551037 & 0.9238966184 & 1.1320630670 & 1.1827963304 \\
\hline & 20 & 0.6686545657 & 0.9260886593 & 1.1175466079 & 1.1626011550 \\
\hline & $\infty$ & $1.04(3)$ & $1.00(3)$ & $0.9999(1)$ & $1.0001(2)$ \\
\hline & $\omega$ & $1^{*}$ & $1.11^{*}$ & 1 & 1.0032 \\
\hline \multirow[t]{11}{*}{$d=0.30$} & $L$ & 0.20 & 0.30 & 0.50 & 0.75 \\
\hline & 6 & 0.3393736439 & 0.5565119224 & 0.8448999662 & 1.0205743378 \\
\hline & 8 & 0.3708396385 & 0.6016754416 & 0.8664772095 & 1.0164775547 \\
\hline & 10 & 0.4001274702 & 0.6385279575 & 0.8836745934 & 1.0137328840 \\
\hline & 12 & 0.4287823558 & 0.6698979484 & 0.8975656827 & 1.0118226252 \\
\hline & 14 & 0.4562391902 & 0.6967097472 & 0.9088380138 & 1.0104117821 \\
\hline & 16 & 0.4820573931 & 0.7197719798 & 0.9180744129 & 1.0093198954 \\
\hline & 18 & 0.5060692639 & 0.7397682858 & 0.9257311017 & 1.0084452275 \\
\hline & 20 & 0.5282757026 & 0.7572450703 & 0.9321538891 & 1.0077262607 \\
\hline & $\infty$ & $0.96(4)$ & $1.0001(1)$ & $1.0000(3)$ & $0.99999(3)$ \\
\hline & $\omega$ & 1.085 & 1.074 & 1 & 1 \\
\hline
\end{tabular}

\begin{tabular}{|c|c|cccc|}
\hline$d=0.50$ & $L$ & 0.30 & 0.40 & 0.50 \\
\cline { 2 - 5 } & 8 & 0.2634463944 & 0.3594043077 & 0.4362871259 & 0.99 \\
& 8 & 0.3071022508 & 0.4156753699 & 0.4975263719 & 0.5485668186 \\
& 10 & 0.3480300086 & 0.4649432503 & 0.5909715317 & 0.6582403419 \\
& 12 & 0.3861353468 & 0.5076282318 & 0.6264865185 & 0.7416603392 \\
& 14 & 0.4210763888 & 0.5445274246 & 0.6565615085 & 0.7702258735 \\
& 16 & 0.4528601529 & 0.5765616531 & 0.6823105808 & 0.8121848167 \\
& 18 & 0.4816890281 & 0.6045559890 & $0.996(2)$ & 1.067 \\
\hline
\end{tabular}

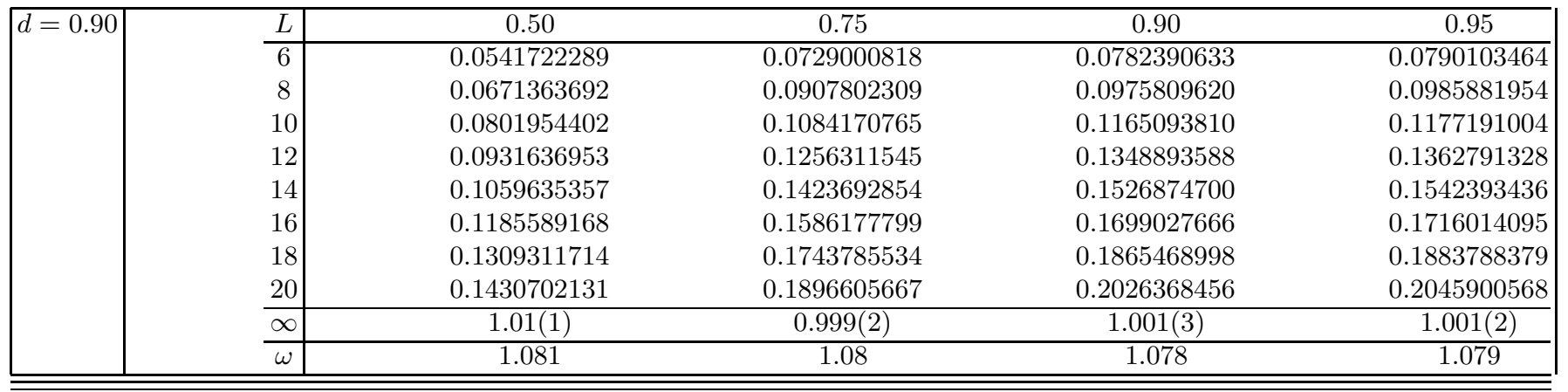


TABLE II. Estimates for the normalized amplitude $A_{L}^{(\mathrm{F})}=L^{2} \Gamma_{L} /\left(\pi^{2} d\right)$ for several values of $p$ and $d$ and free boundary conditions, and the $L \rightarrow \infty$ extrapolation. When the DMRG algorithm did not yield stable results the corresponding finite-size entries are left empty.

\begin{tabular}{|c|c|c|c|c|}
\hline & & \multicolumn{3}{|c|}{$d$} \\
\hline \multirow[t]{12}{*}{$p=0.40$} & $L$ & 0.333 & 0.50 & 0.666 \\
\hline & $\overline{12}$ & 0.8572577379 & 0.6725747038 & 0.4583935580 \\
\hline & 14 & 0.8737590556 & 0.7053840790 & 0.4981815051 \\
\hline & 18 & 0.8978251485 & 0.7547785176 & 0.5630353770 \\
\hline & 20 & 0.9068361253 & 0.7738292422 & 0.5897524204 \\
\hline & 22 & 0.9144401079 & 0.7901798864 & 0.6134826888 \\
\hline & 26 & 0.9265447049 & 0.8167680285 & 0.6537455074 \\
\hline & 28 & 0.9314335748 & 0.8277177891 & 0.6709574987 \\
\hline & 30 & 0.9357318117 & 0.8374491423 & 0.6865761177 \\
\hline & 32 & 0.9395733083 & 0.8461522447 & 0.7008078882 \\
\hline & 34 & 0.9429388328 & 0.8539906968 & 0.7138314424 \\
\hline & $\infty$ & $0.999(3)$ & $0.9999(5)$ & $0.9995(9)$ \\
\hline \multirow{11}{*}{$p=0.50$} & 14 & 0.9239698644 & 0.7686830752 & 0.5656063523 \\
\hline & 16 & 0.9327349799 & 0.7919832846 & 0.5996949840 \\
\hline & 18 & 0.9397547158 & 0.8110625710 & 0.6289496021 \\
\hline & 20 & 0.9454946130 & 0.8269700162 & 0.6543147959 \\
\hline & 22 & 0.9502701952 & 0.8404325820 & 0.6765067894 \\
\hline & 24 & 0.9543020404 & 0.8519708198 & 0.6960780276 \\
\hline & 26 & 0.9577542440 & 0.8619676543 & 0.7134610277 \\
\hline & 28 & 0.9607290383 & 0.8707097768 & 0.7289990177 \\
\hline & 30 & 0.9633226275 & 0.8784202612 & 0.7429679437 \\
\hline & 32 & & 0.8852717697 & 0.7555906209 \\
\hline & 34 & & 0.8913924472 & 0.7670514417 \\
\hline
\end{tabular}

\begin{tabular}{|c|c|c|c|c|c|}
\hline \multirow{10}{*}{$d=0.90$} & $\overline{12}$ & 0.1863167451 & 0.2042528770 & 0.2172237514 & 0.2255705980 \\
\hline & 16 & 0.2362199311 & 0.2576333440 & 0.2728737999 & 0.2824706979 \\
\hline & 18 & 0.2592237747 & 0.2820034649 & 0.2981179611 & 0.3081867029 \\
\hline & 20 & 0.2810372384 & 0.3049794681 & 0.3218249399 & 0.3322848660 \\
\hline & 26 & 0.3400759780 & 0.3665336490 & 0.3849046790 & 0.3961552283 \\
\hline & 28 & 0.3578455055 & 0.3848833645 & 0.4035899034 & 0.4150046251 \\
\hline & 30 & 0.3748295723 & 0.4022778186 & 0.4212504431 & 0.4327914966 \\
\hline & 32 & 0.3908774672 & 0.4187863074 & 0.4378639789 & 0.4495863889 \\
\hline & 34 & 0.4062441268 & 0.4344650723 & & \\
\hline & $\infty$ & $0.986(20)$ & $0.98(3)$ & $0.983(15)$ & $0.984(15)$ \\
\hline
\end{tabular}

